\title{
A Case of Actinic Keratosis of the Scalp (Re)Treated With Ingenol Mebutate $150 \mathrm{Mcg} / \mathrm{G}$.
}

\author{
M Mazzeo, A Dattola*, R Saraceno, L Bianchi \\ Department of Dermatology University of Rome "Tor Vergata", Rome, Italy
}

Received: July 11, 2016; Accepted: July 25, 2016; Published: August 05, 2016

*Corresponding author: Annunziata Dattola, Md university of Rome "Tor Vergata, Department Of Dermatology Policlinico Tor Vergata Viale Oxford, Rome, Italy , 81 00133, Tel. +390620902743; Fax+390620902742; E-mail: Nancydattola@Gmail.Com.

\begin{abstract}
Actinic keratosis (AK) is a cutaneous intraepithelialneoplasma appearing within areas referred as 'fields of cancerization'caused by sun damage progresses to Squamous Cell Carcinoma (SCC). Ingenolmebutate gel is a new effective topical therapy for AK, used once daily for 2 or 3 days depending on the location of lesions. We report a case of patients with multiple AK of the scalp treated for two times with ingenolmebutate $150 \mathrm{mcg} / \mathrm{g}$ gel.
\end{abstract}

Keywords: Actinic keratosis; Scalp; Sun Damage; Ingenolmebutate;

\section{Introduction}

Actinic keratosis or solar keratosis is a common skin lesion caused by sun damage that progresses to squamous cell carcinoma (SCC) [1]. Risk factors for the development of actinic keratosis include fair skin or light pigmentation, Caucasian individuals, freckles, light coloured eyes (blue or green), blonde or red hair, male gender, older age, severe baldness, skin wrinkling, and increased sun exposure due to outdoor occupation/activities. In particular, long-term exposure to ultraviolet light is considered to be a risk factor [2]. For these reasons, lesions are primarily found on sun-exposed areas such as the face, ears, and hands. Clinically, lesions appear scaly, flat, or elevated and may range in colour from red to brown. Actinic keratosisis usually the first lesion in a disease continuum that progresses to invasive SCC. Today, many authors consider actinic keratoses to be real SCCs rather than simple precancerous lesions [1-2]. The risk of transformation is enhanced in patients with increased solar damage, immune suppression, and those of advanced age. Disease progression depends on a number of clinical features such as lesion size, the amount of ulceration, bleeding and in duration, enlargement in diameter, and erythema. Studies have shown that patients with actinic keratosis that have an increased risk of malignancy show lesions that are in durated, inflamed, large (diameter greater than $1 \mathrm{~cm}$ ), rapidly enlarging, bleeding, erythematous, and ulcerated. Ingenolmebutate is an active compound found in the sap of the Euphorbia peplums plant that is known for its dermatological uses, including the treatment of cancerous lesions [3]. This medication treats actinic keratosis lesions by rapidly inducing cell death. The exact mechanism by which this occurs is unknown; however, it is thought that the active ingredient has pleiotropic effects that inhibit tumour cell growth or induce tumour cell death via multiple mechanisms. This may include an immune-mediated response such as protein kinase $\mathrm{C}$ or neutrophil activation. These inflammatory responses may also contribute to the wound-healing properties of the drug. The application of the gel results in the rapid destruction of actinic keratosis lesions. The results of two clinical studies of the biological effects of ingenolmebutate have shown that topical administration induces epidermal necrosis and a deep inflammatory response both in the epidermis and in the upper part of the dermis of the treated skin, with a predominance of infiltration of T cells, neutrophils, and macrophages. The necrosis of the dermis, however, is rarely observed [4]. The efficacy and safety of Picato $150 \mathrm{mg} / \mathrm{g}$, administered on the face and scalp for 3 consecutive days, have been studied in two clinical studies (double-blind, vehicle-controlled) comprising 547 patients (277 in the drug group and 270 in the vehicle group) [5]. Patients were followed for 8 weeks during which they returned for clinical and safety monitoring. Efficacy, measured as the complete and partial clinical cure rate and median percentage reduction, was assessed on day 57 . The results were as follows: $42.2 \%$ of patients treated with the drug $(\mathrm{n}=277)$ obtained a complete clinical cure rate, while $63.9 \%$ achieved partial clinical healing. Further, 3.7\% of all patients treated with the vehicle $(n=270)$ achieved complete clinical recovery, while $7.4 \%$ of patients treated only with the vehicle achieved partial clinical recovery. In the double-blind, placebo-controlled vehicle, up to two cycles of treatment with Picato $150 \mathrm{mg} / \mathrm{g}$ were administered to 450 patients with four to eight actinic keratoses in a $25 \mathrm{~cm}^{2}$ area on the face or scalp. The patients in which a first cycle of treatment did not lead to the complete healing of all actinic keratoses in the area of treatment after 8 weeks were randomised for a further cycle of treatment with Picato or with the vehicle. Patients in which the first cycle of treatment led to full recovery were evaluated at 26 and 44 weeks and randomised for a second course of treatment if they had relapsed in the field. In all patients, efficacy was evaluated at 8 weeks after randomisation. The results were read at 8 and 12 weeks after randomisation: of all patients treated with the drug, $47 \%$ showed the resolution of lesions by the 8 th control week, 
while $18 \%$ were resolved by the 12 th week. Ingenolmebutate is formulated in two different formulations: a $150 \mathrm{mcg} / \mathrm{g}$ gel and a $500 \mathrm{mcg} / \mathrm{g}$ gel. The former is used to treat localised lesions on the face and scalp, while the second is used to treat actinic keratoses localised on the rest of the body. The formulation also changes the administration time, which is 3 consecutive days applying the medication once a day in the first case, while 2 days with daily application in the second case [6]. Ingenolmebutate offers some important advantages, including shorter therapy duration than other drugs, faster healing than rivals on the market, and a favourable side effects profile.

\section{Case Report}

Two months ago, a 74-year-old patient, pho type II, came under our observation at the Department of Dermatology of the University of Rome Tor Vergata. The patient had been chronically exposed to sources of ultraviolet rays throughout life. He showed no significant comorbidities despite an SCC located on the scalp completely being excised 2 years earlier with the endorsement of a skin graft removed from the right forearm. Despite the patient receiving numerous recommendations during these two years, he did not use any photo protection, being exposed quietly to important sources of solar radiation. During the first visit, the patient presented more than 15 actinic keratoses scattered on a cancerous field spread across the entire scalp (FIG.1). In particular, two of these injuries caught our attention because of their hyperkeratinisation and infiltration, fearing that it may instead be two real SCCs, as it is difficult to distinguish actinic keratosis from SCC. Despite our concern, we



considered it suitable to proceed to therapy owing to the future possibility of surgical intervention on a much cleaner area than before. However, an ultrasound of the lymphnode stations and a TC total body excluded a systemic dissemination. Given the important hyperkeratinisation of the lesions, we proceeded with topical therapy based on salicylic acid (20\% for 10 days). We then proceeded with an application of the drug for 3 consecutive evenings, as it gives technical indications. However, given the size of the picture, we treated again the patient's. Further, instead of stopping at a single cycle, we used two cycles with a short break of 10 days between one application and another. In the first inspection, carried out 10 days after the first 3-day cycle, we observed a major improvement; in part, however, this masked the underlying erythema caused by the therapy. In the second inspection carried out 10 days after the second cycle (36 days from the first evaluation), we observed a complete resolution of the picture (FIG 2). With the exception of a slight hyperaemia immediately following the drug application, we observed no significant side effects.

\section{Conclusion}

This case has shown the efficacy and safety of retreatment with ingenolmebutate with clearance of AK lesions present on the scalp. Our experiences adds that ingenol mebutate can be used for field treatment of AK, with a high sustained clearance rate, shorter duration of use and faster resolution of lesions.

\section{References}

1. Lalji A, Khiroya N, Lalji M. Actinic Keratosis and Squamous Cell Carcinoma. Clin Res Dermatol Open Access. 2014;1(1):1-3.

2. Salasche SJ. Epidemiology of actinic keratoses and squamous cell carcinoma. J Am Acad Dermatol. 2000;42(1 Part 2):4-7.

3. Parsippany NJ. Ingenolmebutate (Picato) gel. Leo Pharma Inc. 2012.

4. Rosen RH, Gupta AK, Tyring SK. Dual mechanism of action of ingenolmebutate gel for topical treatment of actinic keratoses: Rapid lesion necrosis followed by lesion-specific immuneresponse.J Am Acad Dermatol. 2012;66(3):486-493. doi: 10.1016/j.jaad.2010.12.038.

5. Siller G, Gebauer K, Welburn P, Katsamas J, Ogbourne SM. PEP005 (ingenolmebutate) gel, a novel agent for the treatment of actinic keratosis: results of a randomized, double-blind, vehicle-controlled, multicentre, PhaseII a study. Australas J Dermatol. 2009;50(1):16-22. doi: 10.1111/j.1440-0960.2008.00497.x.

6. Lebwohl M, Swanson N, Anderson LL, Anita Melgaard, Zhenyi Xu, Brian Berman. Ingenolmebutate gel for actinic keratosis. N Engl J Med. 2012;366(11):1010-1019. doi: 10.1056/NEJMoa1111170. 